\title{
Personalidad y respuesta psicológica en deportistas. Representación temporal y adaptativa del proceso persona-deporte \\ Personality and psychological response in athletes. Temporal and adaptive representation of the person- sport process \\ *Juan González Hernández, **Alexandra Valadez Jiménez \\ *Universidad de Murcia (España), **Universidad de Guadalajara (México)
}

Resumen. Introducción. La identificación de las personas en un contexto de aprendizaje, contempla que sus rendimientos se consideren como parte de la construcción de su personalidad dentro de un proceso constantemente interactivo. En el ámbito deportivo, el deportista contempla tal interacción en un proceso adaptativo donde deben tenerse en cuenta variables psicológicas internas como externas. El presente estudio persigue ahondar en la variabilidad individual y contextual, teniendo en cuenta diferencias y relaciones, que a modo de esfuerzos adaptativos, influyen en la respuesta psicológica. Método. La muestra son deportistas ( $\mathrm{N}=589$ ), con una media de 23.96 años, elegida aleatoriamente y distribuida por género (hombres=64.8\%; mujeres $=35.2 \%$ ), categoría (individual $=35.1 \%$; colectivo=64.8\%) y nivel de deporte (profesionales=41.6\%; amateur=58.4\%). Los instrumentos utilizados han permitido analizar información sobre variables de personalidad, orientación motivacional y estilos de afrontamiento emocionales. Resultados. Diferencias individuales en cuanto a la edad se clasifican en todas las variables; se señalan indicadores más bajos en afabilidad y orientación motivacional (ego) en deportes individuales, de apertura mental y orientación motivacional (tarea) en deportes colectivos, no mostrándose diferencias en coping según el tipo de deporte. Se evidencian correlaciones importantes entre personalidad, coping y motivación. Además, el árbol decisional establecido señala que la energía y la afabilidad son los indicadores que más se diferencian en cuanto a la edad, teniendo en cuenta además la apertura mental, orientación al ego y autocrítica. Conclusiones. Con ello, conocer la variabilidad individual y estrategias de adaptación motivacional y emocional, permiten ajustar en todo momento dicho proceso, teniendo en cuenta los componentes más temperamentales del individuo.

Palabra clave. Deportistas, personalidad, motivación, estilos de afrontamiento, autoeficacia.

Abstract. Introduction. People's identification within a learning context considers their attainments as part of the construction of their personality in a constantly interactive process. In sports, athletes contemplate such an interaction in an adaptive process that should take into account internal and external psychological variables. This study seeks to delve into individual and contextual variability, taking into account differences and relationships as adaptive efforts influencing psychological response. Method. The sample was composed by athletes ( $\mathrm{N}=589)$ with an average age of 23.96 years old, randomly selected and distributed by gender (male $=64.8 \%$; women $=35.2 \%$ ), category (single $=35.1 \%$; group $=64.8 \%$ ) and sport level (professional $=41.6 \%$; amateur $=58.4 \%$ ). The instruments used permitted to analyze information on personality variables, motivational counseling and emotional coping styles. Results. Individual differences by age are classified in all variables; the lowest indicators of affability and motivational orientation (ego) appeared in individual sports, whereas in team sports the lowest scores were found in open-mindedness and motivational orientation (task). No differences by sport type were highlighted in coping. In addition, an established decisional tree indicates that energy and affability are the indicators that differ the most in terms of age, including open-mindedness, self-criticism, and ego orientation, as well. Conclusions.

\section{Introducción}

Las personas se muestran diferentes por razón de la herencia, intereses, motivaciones, ritmos de aprendizaje, grupo social de procedencia, sexo, expectativas vitales, capacidades sensoriales, motrices o

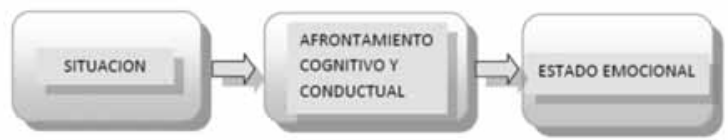

Figura 1. Procesos adaptativos como proceso y resultado

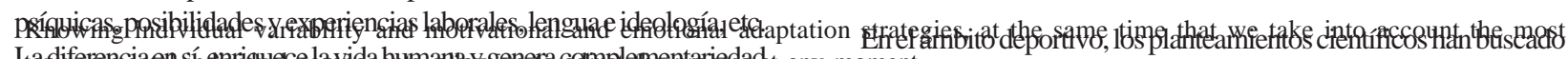

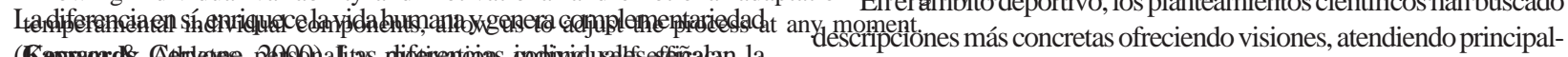

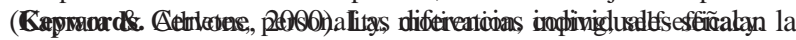
forma de interpretación psicológica y reactividad de la respuesta ante las circunstancias, atendiendo a los elementos que aporta la experiencia adquirida y los más asociados al carácter del individuo.

Se considera la personalidad entonces, como un sistema psicológico que emerge de la interacción-adaptación entre individuo y ambiente, media entre el funcionamiento intrapsíquico (autopercepción, autoestima, autoconocimiento y autorregulación) y evoluciona permanentemente con el medio (Caprara, Vecchione, Barbaranelli, \&Alessandri, 2012; Caprara, Alessandri, \& Eisenberg, 2012; Friedman \& Schustack, 2011).

Las estrategias de afrontamiento, como mecanismos psicológicos para la adaptación a situaciones, traducidos en esfuerzos cognitivos y conductas observables que cada individuo pone en juego de una forma más o menos regular para manejar demandas específicas, externas e internas, contemplado por estudios en el ámbito de la salud (Larsen \& Buss, 2005; Olivari \& Urra, 2007; Romero, 2015) educativos (Rocabert, Gómez, \& Descals, 2006) o deportivos (Gucciardi, 2010).

Adaptarse a las situaciones significa tanto buscar siempre la mejora continua, como que empeore lo menos posible la autoimagen, las relaciones interpersonales, la afectividad y el bienestar. mente a diferencias de género y edad(González, Garcés de los Fayos, \& Ortega, 2014; Li, Lee, \& Solmon, 2006), aunque dando poca importancia a los niveles de dedicación, entre otras características psicosociales.

Tal y como indican algunos autores en diversos planteamientos científicos en el ámbito deportivo, se ha encontrado una estrecha relación entre la autoeficacia y la motivación (Treasure et al., 2001), el esfuerzo (Tenenbaum et al., 2005), la constancia (Guillén Rojas, 2012) o la autorregulación emocional (Willians \& DeSteno, 2008), dirigidas a las actividades que realizan los deportistas. Viene a dar sentido entonces a la conexión entre el conocimiento y la acción, en el que lo cognitivo media a través de lo que se denomina pensamiento autorreferente (Bandura, 2001).

Por todo ello, y puesto que la práctica deportiva constantemente requiere la adaptación hacia un camino de superación de objetivos (Bertollo, Saltarelli, \& Robazza, 2009), fortaleza mental (Kuan \& Roy, 2007; Jones, 2002), perfiles de logro (Mendez-Giménez, González, Cechinni, \& Fernández-Río, 2013; Álvarez, Esteban, Falcó, HernándezMendo, \& Castillo, 2014; Pérez, 2015), incluso de experimentación de orgullo (Willians \& DeSteno, 2008), deben tenerse en cuenta cómo las características individuales y sus relaciones permiten o influyen en el citado proceso adaptativo.

Los objetivos del presente trabajo se dirigen a describir diferencias y correspondencias en una muestra de deportistas en personalidad y estrategias de afrontamiento motivacional y emocional, controlando las variables género, tipo de deporte y nivel deportivo de los participantes. 


\section{Método}

\section{Participantes}

El estudio se distribuye en una muestra de 589 deportistas, seleccionada de forma aleatoria, sobre diferentes modalidades deportivas y sin criterio alguno de exclusión. La descripción se realiza a través del género (64.8\% hombres-35.2\% mujeres), y edad ( $\mathrm{M}=23.96$; $\mathrm{DT}=$ 5.9), distribuidos en cuatro rangos de edad (tabla 1). Las variables contextuales a tener en cuenta son aquellas que tiene relación directa con el deporte practicado, tanto en tipo (individual 32.58\%-colectivo 67.16\%) como en el nivel del mismo (profesional 42.6\%-amateur 58.4\%).

\section{Materiales/instrumentos}

Personalidad. Se ha utilizado la adaptación al castellano del cuestionario Big Five (BFQ; Bermúdez, 1998. Cuestionario Big Five. $2^{\mathrm{a}}$ Edición. Adaptación al castellano del Cuestionario BFQ de Caprara et al., 1993), compuesto por 132 items, distribuidos en una escala likert de 1 («completamente falso») a 5 («completamente verdadero»). Describiendo escalas de Energía, Afabilidad, Tesón/Responsabilidad, Estabilidad Emocional y Apertura Mental, mostrando un índice de consistencia interna para la muestra de á=.87.

Orientación motivacional. Para esta medida, se utiliza la versión española del Perception of Success Questionnaire (POSQ; Roberts, Treasure, \& Balagué, 1998), que consta de 12 ítems, 6 para el factor tarea $(3,4,7,8,10$ y 11) y 6 para el factor ego (1, 2, 5, 6, 9 y 12). Tiene un formato escala Likert de cinco puntos donde 1 significa «muy en desacuerdo» y 5 «muy de acuerdo» para cada elemento. La pregunta inicial es «en mi práctica deportiva tengo éxito cuando...». La fiabilidad del instrumento es aceptable ya que se muestra con un valor alpha de cronbach de .84 para la subescala ego y de .81 para la subescala tarea respectivamente.

Estrategias de afrontamiento. Se utilizó el Cuestionario deEstilos de Afrontamiento (CSI; Cano, Rodríguez, \& García Martínez, 2007), que en su parte cuantitativa, consta de 41 ítems, distribuidos en una escala likert de 0 (en absoluto) a 4 (totalmente), de los cuales 40 se encuentran directamente relacionados con las escalas de afrontamiento (Resolución de problemas, Autocrítica, Expresión emocional, Apoyo social, Pensamiento desiderativo, Evitación de problemas, Reestructuración cognitiva y Retirada social) y 1 directamente relacionado con el grado de Eficacia percibida del afronta- miento. El índice de consistencia interna alpha de cronbach para la muestra de .80 .

\section{Procedimiento}

Se sigue un mismo protocolo de actuación diseñado: (1) primera visita para explicación de la investigación y solicitud de autorizaciones y/o consentimientos informados necesarios; (2) citación grupal de deportistas; (3) breve explicación del protocolo a seguir; (4) facilitar una batería de cuestionarios completa y numerada que contestaban individualmente, junto con un consentimiento informado. En todo momento de la recogida muestral se encuentra presente un evaluador para cualquier duda o aclaración. El análisis se ha realizado con el programa estadístico SPSS. 21.0., obteniéndose medidas descriptivas (frecuencias), homogeneidad y fiabilidad, ANOVA, análisis discriminantes y de correlación, y modelo de clasificación-predicción a través de árbol de decisiones (según la edad del deportista).

\section{Resultados}

\section{ANOVA según el rango de edad}

La tabla 2 refleja el análisis de las diferencias en la distribución por rango de edad, se muestra que existen diferencias estadísticamente significativas entre los deportistas según su grupo de edad en la escala Energía $\left(\mathrm{F}_{3,586}=7.143 ; p=.014\right)$ para los más jóvenes, y Apertura Mental $\left(\mathrm{F}_{3,586}=11.026 ; p=.00\right)$, Tesón $\left(\mathrm{F}_{3,586}=12.731 ; p=.03\right) \mathrm{y}$ Estabilidad Emocional $\left(\mathrm{F}_{3,586}=6.382 ; p=.00\right)$ para el grupo de los más experimentados. No encontrando valores estadísticamente significativos en Afabilidad $\left(\mathrm{F}_{3,586}=1.087 ; p=.354\right)$.

\begin{tabular}{|c|c|c|c|c|c|c|c|c|c|}
\hline \multicolumn{10}{|c|}{ Distribución según rangos de edad de los deportistas } \\
\hline & \multicolumn{2}{|c|}{ menos 19 años } & \multicolumn{4}{|c|}{$88(14.1 \%)$} & \multicolumn{3}{|c|}{14,1} \\
\hline \multicolumn{3}{|c|}{ entre 20 años- 29 años } & \multicolumn{4}{|c|}{$458(73.5 \%)$} & \multicolumn{3}{|c|}{87,6} \\
\hline entre 3 & \multirow{2}{*}{\multicolumn{2}{|c|}{$\frac{30 \text { años-35 años }}{\text { más de } 35 \text { años }}$}} & \multirow{2}{*}{\multicolumn{4}{|c|}{$\begin{array}{l}51(8.2 \%) \\
26(4.2 \%)\end{array}$}} & \multicolumn{3}{|c|}{95,8} \\
\hline & & & & & & & & 100 & \\
\hline \multicolumn{10}{|l|}{ Total $=589$} \\
\hline \multicolumn{10}{|c|}{$\begin{array}{l}\text { Tabla } 2 . \\
\text { Análisis de Varianza (ANOVA) de las dimensiones para la variable rango de edad de los } \\
\text { deportistas }\end{array}$} \\
\hline & \multicolumn{2}{|c|}{$\begin{array}{c}<19 \text { años } \\
(\mathrm{n}=142)\end{array}$} & \multicolumn{2}{|c|}{$\begin{array}{l}\text { Entre } 20 \text { y } 29 \\
\text { años }(n=327)\end{array}$} & \multicolumn{2}{|c|}{$\begin{array}{c}\text { Entre } 30 \text { y } 35 \\
\text { años }(n=84)\end{array}$} & \multicolumn{2}{|c|}{$\begin{array}{c}>\text { de } 35 \text { años } \\
(\mathrm{n}=36)\end{array}$} & \multirow[t]{2}{*}{$F(p)$} \\
\hline Personalidad & $M$ & $D T$ & $M$ & $D T$ & $M$ & $D T$ & $M$ & DT & \\
\hline Energía & 87.74 & 11.42 & 79.99 & 10.69 & 79.33 & 9.69 & 83.69 & 10.18 & $7.143(.04)^{*}$ \\
\hline Afabilidad & 84.13 & 11.02 & 82.12 & 10.82 & 81.76 & 10.27 & 83.65 & 7.57 & 14.354 \\
\hline Tesón & 82.22 & 8.92 & 81.43 & 10.77 & 80.92 & 10.43 & 87.50 & 11.27 & $12.731(.03)^{*}$ \\
\hline $\begin{array}{l}\text { Estabilidad } \\
\text { Emocional }\end{array}$ & 68.03 & 11.90 & 70.44 & 11.82 & 71.33 & 13.36 & 78.62 & 12.24 & $6.382(.00)^{* *}$ \\
\hline Apertura Mental & 77.44 & 9.32 & 80.12 & 10.24 & 79.18 & 10.09 & 85.81 & 11.28 & $11.026(.00)^{*+}$ \\
\hline \multicolumn{10}{|c|}{ Afrontamiento motivacional } \\
\hline Percepción ego & 26.14 & 4.35 & 24.06 & 4.84 & 20.83 & 2.35 & 21.19 & 4.68 & $5.793(.03)^{*}$ \\
\hline Percepción tarea & 26.02 & 5.64 & 25.73 & 3.59 & 23.46 & 5.17 & 24.37 & 5.26 & .428 \\
\hline \multicolumn{10}{|c|}{ Afrontamiento cognitivo-emocional } \\
\hline $\begin{array}{l}\text { Resolucion de } \\
\text { problemas }\end{array}$ & 16.5 & 2.12 & 15.66 & 2.79 & 15.90 & 2.92 & 16.27 & 2.06 & .378 \\
\hline Autocrítica & 12.88 & 3.87 & 12.83 & 3.06 & 12.76 & 3.90 & 11.12 & 2.98 & .101 \\
\hline $\begin{array}{l}\text { Expresion } \\
\text { Emocional }\end{array}$ & 13.86 & 3.04 & 13.34 & 3.18 & 13.00 & 2.00 & 12.19 & 3.38 & .159 \\
\hline $\begin{array}{l}\text { Pensamiento } \\
\text { Desiderativo }\end{array}$ & 14.30 & 3.42 & 14.25 & 3.14 & 13.61 & 2.99 & 13.31 & 3.76 & .364 \\
\hline Apoyo Social & 15.69 & 3.19 & 15.19 & 3.95 & 14.16 & 2.23 & 14.81 & 3.24 & .058 \\
\hline $\begin{array}{l}\text { Reestructuracion } \\
\text { Cognitiva }\end{array}$ & 10.32 & 3.82 & 14.63 & 3.11 & 14.41 & 2.80 & 15.27 & 2.90 & .275 \\
\hline $\begin{array}{l}\text { Evitacion de } \\
\text { Problemas }\end{array}$ & 11.07 & 2.66 & 11.83 & 3.79 & 10.82 & 3.77 & 11.08 & 3.45 & $14.372(.03)^{*}$ \\
\hline Retirada Social & 14.14 & 2.59 & 11.38 & 3.36 & 11.43 & 2.21 & 10.42 & 3.49 & .057 \\
\hline
\end{tabular}

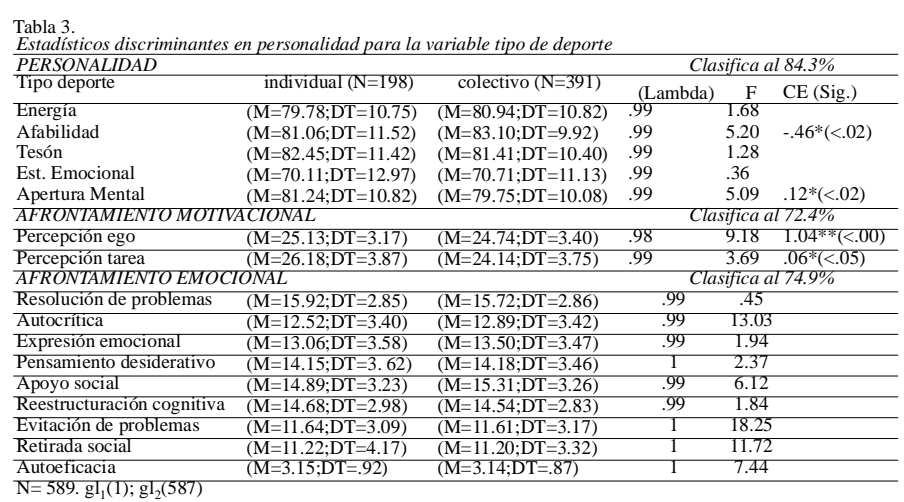

En cuanto a la orientación motivacional, se señalan diferencias significativas tanto a favor de la orientación hacia el ego $\left(\mathrm{F}_{3,586}=5.793 ; p\right.$ $=.03$ ) con respecto a los más jóvenes, no apareciendo diferencias significativas sobre la orientación hacia la tarea.

Las estrategias de afrontamiento han mostrado que únicamente en la dimensión evitación de problemas existen diferencias significativas $\left(F_{3,586}=14.372 ; p=.03\right)$ en el grupo de los más jóvenes, no siendo de relevancia en el resto de estrategias de afrontar conflictos emocionales.

\section{Análisis discriminantes según el tipo de deporte}

Para las medias de la muestra en la variable tipo de deporte (tabla 3), los indicadores más altos se aprecian en tesón para deportes individuales y afabilidad para deportes colectivos. Los análisis univariantes señalan discriminantes adecuados (Lambda $=.96 ; X^{2}=23.30 ; p<.00$ ), señalando funciones significativas hacia afabilidad en deportes colectivos y apertura mental a favor de deportes individuales, reclasificando correctamente un $84.2 \%$.

Observando los indicadores en las escalas de motivación, los que practican deportes individuales señalan índices más altos tanto en percepción de ego como en percepción de tarea. La relación univariante (Lambda = .98; $X^{2}=9.12 ; p<.01$ ), muestra que los practicantes de deportes individuales discriminan significativamente hacia la percepción de ego $(<.00)$ y los que practican deportes colectivos en percepción de tarea $(<.05)$, reclasificando correctamente un $72.4 \%$. 
Conrespecto a las estrategias de afrontamiento emocional, la muestra presenta entre los valores más altos en resolución de problemas y los más bajos de retirada social tanto en deporte individual y colectivo. La función discriminante, en este caso, no señala orientaciones significativas en cuanto al tipo de deporte practicado.

\section{Correlaciones}

Las relaciones bilaterales entre las variables se muestran significativas destacando las directas de energía con orientación al ego ( $\mathrm{r}=$.714), tesón con autoeficacia $(\mathrm{r}=.730)$ y orientación al ego $(\mathrm{r}=.672)$, y apertura mental con resolución de problemas $(\mathrm{r}=.774)$ y reestruturación cognitiva $(\mathrm{r}=$.757). De forma contraria, también se reflejan relaciones inversas entre energía con retirada social ( $\mathrm{r}=-.422)$, afabilidad con autocrítica $(\mathrm{r}=-.725)$, tesón con retirada social $(\mathrm{r}=-.748)$, y apertura mental con la retirada social $(\mathrm{r}=$-.607) y con orientación al ego $(r=$ .416) de energía ( $\mathrm{F}$ = 12.43; Nódulo 1), por lo que se mostrarán como más dinámicos, con iniciativa y activos. De ellos, los que menos energía muestran (45.1\%) se clasifican a través de la orientación hacia el ego ( $F$ = 15.08; Nódulos 3, 7 y 8), lo que confiere a esta variable una interesante influencia de la tendencia a distorsionar lo que atrae a los deportistas a ser dominantes, incluso a ser optimistas. Los deportistas que indican medias más altas en apertura mental (39\%), se clasifican a través de los indicadores de afabilidad ( $\mathrm{F}=13.79$; Nódulo 2), de ser cordiales, de buscar lo cooperativo, lo asociativo con otros, lo prosocial; y de los mismos, la tendencia a menor orientación a los demás (menor afabilidad) se observa que clasifica con los que señalan usar la autocrítica (F = 14.34; Nódulos 5, 9 y 10).

\section{Discusión}

Se ha pretendido establecer la relación entre algunas de las cualida-

Tabla 4.

Relaciones lineales entre las diferencias individuales y estrategias de afrontamiento motivacionales y emocionales

\begin{tabular}{|c|c|c|c|c|c|c|c|c|c|c|c|}
\hline & 1 & 2 & 3 & 4 & 5 & 6 & 7 & 8 & 9 & 10 & 11 \\
\hline Energía & $.325^{+*}(.000)$ & $-.493^{*}(.020)$ & $.686^{*}(.032)$ & & $.487^{* *}(.000)$ & $.571^{* *}(.000)$ & $-.479^{* *}(.000)$ & $-.642^{* *}(.000)$ & $.500^{* *}(.000)$ & $.714^{4+*}(.000)$ & $.453^{*+*}(.000)$ \\
\hline Afabilidad & $.207^{+*}(.000)$ & $-.725^{+*+}(.002)$ & $.508^{* *}(.007)$ & & $.346^{* *}(.000)$ & $.285^{+4}(.000)$ & $-.225^{+*}(.000)$ & $-.367^{*+*}(.000)$ & $.169^{*+}(.000)$ & $.334^{-4}(.000)$ & $.132^{2+*}(.001)$ \\
\hline $\begin{array}{l}\text { Tesón/Respons } \\
\text { abilidad }\end{array}$ & $.484^{+*}(.000)$ & $-.494^{*}(.018)$ & $-.508^{+4+1}(.007)$ & & & $.514^{+4+(}(.004)$ & $-.462^{+*}(.000)$ & $-.648^{* *+}(.000)$ & $.730^{* *}() .001$ & $.672^{+1}(.000)$ & $.422^{*+1}(.002)$ \\
\hline $\begin{array}{l}\text { Estabilidad } \\
\text { emocional }\end{array}$ & & $-.485^{* * *}(.000)$ & $-.258^{+*+}(.000)$ & $-.353^{+*}(.000)$ & & $.347^{* *}(.000)$ & $.442^{* *}(.000)$ & & $.360^{* 4}(.000)$ & & $-.325^{* *}(.002)$ \\
\hline $\begin{array}{l}\text { Apertura } \\
\text { mental }\end{array}$ & $.743^{* *}(.000)$ & $.783^{* *}(.000)$ & & & $.672^{* *}(.000)$ & $.567^{* *}(.000)$ & $-.506^{* *}(.000)$ & $-.607^{+*}(.000)$ & $.533^{* 4}(.000)$ & $-.416^{* *}(.000)$ & \\
\hline
\end{tabular}

* significativa si $p<.05$; ** significativa si $p<.01$

1(Resolución de problemas); 2(Autocrítica); 3(Expresión Emocional); 4(Apoyo Social); 5(Pensamiento desiderativo); 6(Reestructuración cognitiva); 7(Evitación de problemas); 8(Retirada social); 9(Autoeficacia); 10(Orientación hacia el ego); 11(Orientación hacia la tarea)

\section{Modelo predictivo a través de árbol de valores}

Tomando como referencia la elaboración de un modelo de relación que permita la agrupación de los participantes, con el propósito de establecer la influencia predictiva de las características de personalidad sobre la edad-experiencia deportiva, se clasifica a través del correspondienteárbol de decisión (figura2), a través del algoritmo CHAID (Román González, \& Lévy, 2003; Fowdar, Bandar, \& Crockett, 2004) ( $p<.05)$, donde se aprecia significativamente que son los deportistas con mayores índices de apertura mental los que se encuentran en el rango de 2029 años de edad ( $\mathrm{F}=15.02$; Nódulo 0). De los mismos, los que señalan medias más bajas (61\%), se clasifican por tener adecuados indicadores

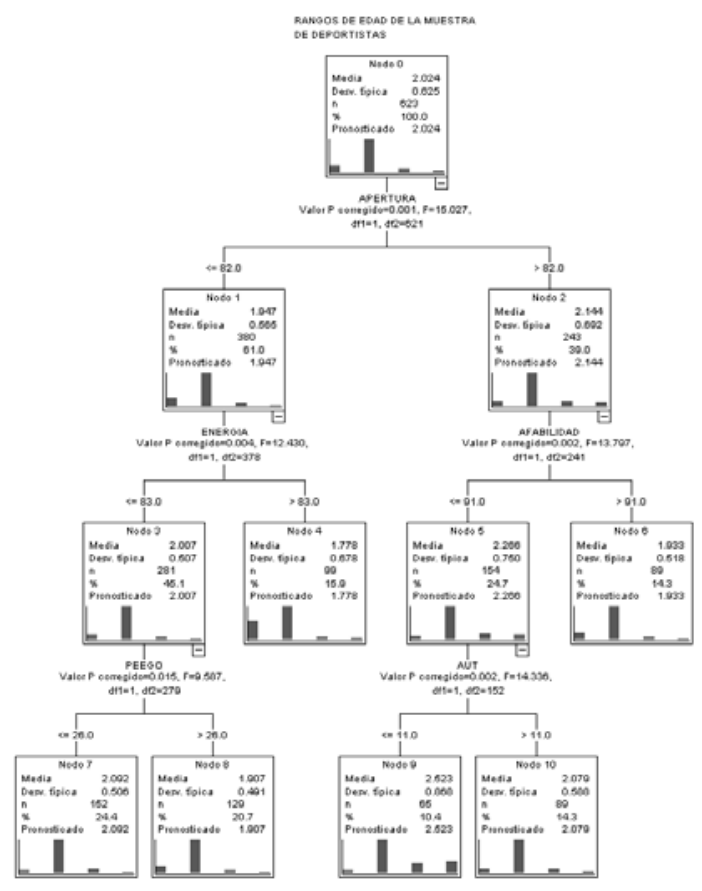

Figura 2. Árbol de decisiones sobre la personalidad y respuesta psicológica, en cuanto a la variable edad-experiencia deportiva des individuales adaptativas que la persona aporta en su práctica deportiva, atendiendo a sus características de personalidad, orientación motivacional y afrontamiento cognitivo-emocional, con un planteamiento de lo general a lo específico de cara a la explicación de las condiciones que permiten a los deportistas alcanzar procesos de experiencia y mantenimiento de la práctica deportiva.

Tal y como se ha dispuesto en el modelo de clasificación planteado, la variable que determina el proceso de adquisición de experiencia y el correspondiente mantenimiento de la práctica que realiza a lo largo de la vida deportiva es la Apertura Mental. En este caso, la orientación hacia el ego pronostica bajos niveles de Energía (más independientes, familiares y rutinarios, más reflexivos y con menos tendencia a la acción), que a su vez predice bajos niveles de Apertura Mental (deportistas más convencionales, poco flexibles en la búsqueda de nuevos planteamientos, prefieren la familiaridad a lo novedoso, son moderados y apegados a la tradición, etc...). Por otro lado, la autocrítica pronostica bajos niveles de Afabilidad (confía poco en otros, presta u ofrece poca ayuda, muestra pocas habilidades empáticas, etc.), que predice altos niveles de Apertura Mental (tienden a buscar nuevas experiencias personales y concibe de una manera creativa su futuro, muestran relación fluida con su imaginación, aprecian el arte y la estética, son consecuentes con sus emociones, prefieren romper con la rutina y suelen poseer conocimientos sobre amplios temas debido a su curiosidad intelectual)

En general, los deportistas indican ser más enérgicos, tenaces, con mayor estabilidad emocional y menores indicadores de apertura mental con respecto a la población general (Bermúdez, 1998). Por este camino, los deportistas analizados se muestran perseverantes, dinámicos, cooperativos y tienen mayor control de sus emociones confirmando planteamientos acerca de algunas de estas características en contextos deportivos (Allen, \& Laborde, 2014; Cantarero, \& Carranque, 2016; González et al., 2014; Khan \&Ahmed, 2014; Ruiz Barquin, 2012), aunque contrastando otros principalmente, en la no existencia diferencial con la mayor apertura mental en deportistas (García-Naveira, Ruiz, \& Pujals, 2011; Hoyt, Rhodes, Hausenblas, \& Giacobbi, 2009).

En cuanto al tipo de deporte, en el deporte individual los deportistas se muestran más tenaces y perseverantes, así como en el deporte colectivo más afables, cordiales y controlan más sus emociones 
(Rhodes \& Smith, 2006); encontrándose diferencias importantes en apertura mental a favor de los practicantes de deportes individuales, apoyando la ya literatura existente acerca de este tipo de estudios (Colzato, Szapora, Pannekoek, \& Hommel, 2013; Eccles \& Wigfield, 2002).

Al observar la orientación motivacional estudiada, se han encontrado que los procesos de motivación son más significativos en los que practican deporte individual, la orientación hacia el ego se muestra principalmente relacionada con indicadores de energía, y las principales diferencias que muestran los deportistas se encuentran entre los menores de 19 años, tanto en motivarse sobre sí mismos como en la evitación de problemas. En este trabajo, la muestra indica lo que numerosa literatura científica sobre las metas de logro ya señala anteriormente, la orientación preocupantemente hacia el ego que genera la práctica deportiva, principalmente derivada de la visión competitiva de la misma (Gutiérrez, 2014; Martín, Tendero, \& Bañuelos, 2006; Standage \& Treasure, 2002).

Cuando el deportista se percibe como altamente capaz para realizar determinada tarea, muestra un gran interés y compromiso, invierte más tiempo y esfuerzo, anticipa resultados, planifica metas, se critica menos (Bossio, Raimundi, \& Correa, 2012); aspectos que le permiten al individuo aumentar el esfuerzo requerido para la tarea y persistir aunque tenga dificultades o adversidades (Jones, Hanton, \& Connaughton , 2007; Kanniyan, George, \& Valiyakath, 2015; Rhodes \& Smith, 2006).

Contando imprescindiblemente con la relación (adecuada-inadecuada, fuerte, débil o nula, de predictibilidad o relación directa) existente entre personalidad y funciones psicológicas, el deportista se adapta permanentemente a situaciones deportivas constantemente cambiantes (Blanchette, Ramocki, O’del, \& Casey, 2005; Caprara \& Cervone, 2002).

\section{Conclusiones}

Plantear la información resultante de estas relaciones como indicadores para la mejora de la práctica deportiva, permitirá al contexto deportivo y al profesional de la psicología deportiva, encontrar nuevas formas de afinar el trabajo psicológico con deportistas, tanto en intervención directa (con los propios deportistas) como en indirecta (asesorando a entrenadores, padres u otros).

La orientación al ego está ligada a un énfasis en las atribuciones de habilidad o capacidad, mientras que la implicación en la tarea corresponde al empleo de las atribuciones de esfuerzo. Es en este caso, cuando se debe aprovechar la información que se obtiene sobre una muestra de referencia, para ajustar las metodologías de enseñanza y transmisión de la práctica deportiva en base a las características de los deportistas, en pos de facilitar la adaptación de sus cualidades a las exigencias de la práctica deportiva que realicen. De esta manera, conocer que los deportistas con menos apertura mental han tenido influencias sobre la necesidad de crecer sobre su deporte en base a ganar a los demás o a ser mejores sobre los resultados que se obtienen, permite reconducir las formas sobre las cualidades de esfuerzo y perseverancia, base de la autorresponsabilidad y de los recursos de apoyo social.

Cuando un deportista aprende a través de la experiencia y de la adquisición de conciencia (proceso autorreferencial) de sus propias cualidades, desarrolla habilidades de autocrítica, de aceptación, de respeto hacia el apoyo social, lo que le confiere una mayor cualidad de aceptar y buscar lo nuevo, lo diferente, lo que le permite crecer y lo que le permite identificarse.

Así mismo, y como propuesta de futuro, es preciso entender el cambio actitudinal a través de planteamientos cognitivo-sociales contemplando modelos teóricos complementarios, explicativamente mucho más específicos que las teorías típicas de la personalidad, aprendizaje o motivación. Aportando de esta manera, mayor información sobre la influencia de la interacción entre algunas variables psicológicas, aspectos de personalidad individuales del deportista e influencias contextuales de cada uno de los ámbitos y niveles de práctica deportiva en la que se encuentran. Permitiendo así la transmisión de la influencia recíproca persona-deporte a través de procesos de enseñanza o entrenamiento más eficaces y ajustados.

\section{Referencias}

Allen, M. S., \& Laborde, S. (2014). The role of personality in sport and physical activity. Current Directions in Psychological Science, 23(6), 460-465.

Álvarez, O., Esteban, I., Falcó, C., Hernández-Mendo, A. y Castillo, I. (2014). Perfil de habilidades psicológicas en taewondistas universitarios y su relación con el éxito en competición. Cuadernos de Psicología del Deporte, 14(3), 13-20.

Bandura,A. (2001). Social Cognitive Theory: An Agentic Perspective. Annual Review Psychology, 52, 1-26.

Bermúdez, L. (1998). Cuestionario Big Five. 2ª Edición. Adaptación al castellano del Cuestionario BFQ de Caprara et al., 1993). Madrid: Tea ediciones.

Bertollo, M., Saltarelli, B., \& Robazza, C. (2009). Mental preparation strategies of elite modern pentathletes. Psychology of Sport and Exercise, 10, 244-54

Blanchette, D. M., Ramocki, S. P., O’del, J. N., y Casey, M. S. (2005). Aerobic exercise and creative potential: Immediate and residual effects. Creativity Research Journal, 17(2-3), 257-264. doi: 10.3389/ fnhum.2013.00824.

Bossio, M. R., Raimundi, M. J., \& Correa, L. G. (2012). Programa de Entrenamiento en Habilidades Psicolgógicas en jugadoras de voleibol de alto rendimiento. Cuadernos de Psicología del Deporte, 12(1), 9-16.

Cano, F.J., Rodríguez, L., \& García Martínez, J. (2007). Adaptación española del Inventario de Estrategias de Afrontamiento. Actas Españolas de Psiquiatría, 35(1), 29-39.

Cantarero, J. C., \& Carranque, G. Á. (2016). Relación entre el pensamiento creativo y el ejercicio físico en personas adultas. Revista Iberoamericana de Psicología del Ejercicio y el Deporte, 11(1), 4752.

Caprara, G.V. \& Cervone, D. (2000). Personality: Determinants, dynamics, and potentials. New York: Cambridge University Press; 2000.

Caprara, G.V.,Alessandri, G., \& Eisenberg, N. (2012). Prosocialidad: La contribución de los rasgos, valores y creencias de autoeficacia. Revista de Personalidad y Psicología Social. 102(6), 1289-1303.

Caprara, G.V., Barbaranelli, C., Borgogni, L., \& Perugini, M. (1993). The «Big Five Questionnaire»: A new Questionnaire to asses the five factor model. Personality and Individual Differences. 15, 28188.

Caprara, G.V., Vecchione, M., Barbaranelli, C., \&Alessandri, G. (2012). Emotional Stability and Affective Self-regulatory Efficacy Beliefs: Proofs of Integration between Trait Theory and Social Cognitive Theory. European Journal of Personality, 27(2), 145-54.

Colzato, L. S., Szapora, A., Pannekoek, J. N., y Hommel, B. (2013).The impact of physical exercise on convergent and divergent thinking. Frontiers in Human Neuroscience, 7, 824. doi: 10.3389/ fnhum.2013.00824.

Eccles, J.S., Wigfield,A. (2002). Motivational beliefs, values, and goals. Annual Review of Psychology, 53, 109-32.

Fleeson, W., Furr, R. M., Jayawickreme, E., Meindl, P., \& Helzer, E. G (2014). Character: The Prospects for a Personality Based Perspective on Morality. Social and Personality Psychology Compass, 8(4), 178-191.

Fowdar, J., Bandar, Z., Crockett, K. (2004). Inducing fuzzy decisión trees in non-deterministic domains using CHAID. En Barr, V. y Markou, Z. Proceedings of the Seventeenth International Florida Artificial Intelligence. Research Society Conference, 838-43. California: AAAI Press.

Friedman, H.W. \& Schustack M.W. (2011). Personalidad: teorías clásicas y la investigación moderna. (5. ${ }^{a}$ ed.). Boston, MA: Allyn \& Bacon.

García-Naveira, A., Ruiz, R. y Pujals, C. (2011). Diferencias en perso- 
nalidad en función de la práctica o no deportiva, nivel de competición y categoría por edad en jugadores de fútbol desde el modelo de Costa y McCrae. Revista de Psicología del Deporte, 20(1), 29-44.

González, J., Garcés de los Fayos, E. J., \& Toro, E. Avanzando en el camino de diferenciación psicológica del deportista. Ejemplos de diferencias en sexo y modalidad deportiva. Anuario de Psicología. 44(1), 31-44.

Gucciardi, D. (2010). Mental toughness profiles and their relations with achievement goals and sportmotivation in adolescent Australian footballers. Journal of Sports Sciences 28(6), 615-25.

Guillén Rojas, N. (2007). Implicaciones de la Autoeficacia en el rendimiento deportivo. Pensamiento Psicológico, 3(9), 21-32.

Gutiérrez, M. (2014). Relaciones entre el clima motivacional, las experiencias en educación física y la motivación intrínseca de los alumnos. Retos. Nuevas Tendencias en Educación Física, Deporte y Recreación, 26, 9-15.

Hoyt, A., Rhodes, R., Hausenblas, H. y Giacobbi, P. (2009). Integrating five-factor model facet-level traits with the theory of planned behavior and exercise. Psychology of Sport and Exercise, 10, 565572.

Jones, G. (2002). What is this thing called mental toughness? An investigation of elite sport performers. Journal of applied sport psychology, 14(3), 205-218.

Jones, G, Hanton, S., \& Connaughton, D. (2007). A framework of mental toughness in the world's best performers. Sport Psychology, 21, 243-64.

Kanniyan, A., George, A., \& Valiyakath, S. (2015). Personality traits: an analytical study between sedentary males and sports males. Ovidius University Annals, Physical Education and Sport/Science, Movement and Health Series, 15(1), 5-9.

Khan, B., \& Ahmed, A. (2014). Profiling Personality Patterns of Champions \& Working Towards Development of the SuccessProne Athletes' Personality Index (SAPI)-A Sports Management Perspective. Pakistan Journal of Statistics, 30(6), 1405-1420.

Kuan, G. \& Roy, J. (2007). Goal profiles, mental toughness and its influence on performance outcomes among Wushu athletes. Journal of Sports Science and Medicine, 6, 28-33.

Larsen, R. \& Buss, D. (2005). Psicología de la personalidad. México: McGraw Hill.

Li, W., Lee, A, \& Solmon, M. (2006). Gender differences in beliefs about the influence of ability and effort in sport and physical activity. Sex Roles, 54, 147-56

Martín, J. J., Tendero, G. R., \& Bañuelos, F. S. (2006). Orientación y clima motivacional, motivacion de logro, atribuición de éxito y diversión en deporte individual. Apunts Educación Física y Deportes,1, 5-11.

Méndez-Giménez, A., González González de Mesa, C., Cecchini Estrada, J.A., \& Fernández-Río, J. (2013). Perfiles motivacionales y sus consecuencias en educación física. Un estudio complementario de metas de logro 2x2 y autodeterminación. Revista de Psicología del Deporte, 22(1), 0029-38.

Olivari, C. \& Urra, E. (2007). Autoeficacia y conductas de salud. Ciencia y Enfermería, 13(1), 9-15

Pérez, A. F. (2015). La caracterización del perfil del deportista de atletismo de alto nivel. Revista de Investigación en Educación, 13(2), 219-243.

Rhodes, R. E. y Smith, N. (2006). Personality correlates of physical activity: a review and meta-análisis. British Journal of Sports Medicine, 40(12), 958-965.

Roberts, G.C., Treasure, D.C., \& Balagué, G. (1998). Achievement goals in sport: The develoment and validation of the Perception of Success Questionnaire. Journal of Sport Sciences, 16, 337-47

Rocabert, E., Gómez, A. \& Descals. A. (2006). Elaboración de un cuestionario de personalidad eficiente para el análisis de la conducta vocacional universitaria. Revista de Estudios e Investigación en Psicología y Educación, 13, 399-412.

Román González, Må.V., \& Lévy Mangin, J.P. (2003). Clasificación y segmentación jerárquica. En Lévy Magin J.P. \& Varela Mallou, J. (Eds) Análisis multivariable para las Ciencias Sociales, (pp.567630). Madrid: Pearson Educación.

Romero, C. E. Meta-análisis del efecto de la actividad física en el desarrollo de la resiliencia: Un meta-análisis. Retos. Nuevas Tendencias en Educación Física, Deporte y Recreación, 28, 98-103.

Ruiz Barquín, R. (2008). Aportaciones del análisis subdimensional del cuestionario de personalidad BFQ para la predicción del rendimiento en judokas jóvenes de competición. Cuadernos de Psicología del Deporte. 8(1), 5-29.

Ruíz Barquín, R. (2012). Relaciones entre características de personalidad y estados de ánimo: un estudio con deportistas de combate universitarios. Revista Iberoamericana de Psicología del Ejercicio y el Deporte, 7(1), 89-112.

Standage, M. \& Treasure, D. (2002). Relationship among achievement goal orientations and multidimensional situational motivation in physical education. British Journal of Educational Psychology, 72, 87-103.

Tenenbaum, G., Lidor, R., Lavyan, N., Morrow, K., Tonnel, S., \& Gershgoren, A. (2005). Dispositional and task-specific socialcognitive determinants of physical effort perseverance. The Journal of psychology, 139(2), 139-158.

Treasure D.C., Duda, J., Hall, H.K., Roberts, G.C., Ames, C., Maehr, M.L. (2001). Clarifying misconceptions and misinterpretations in achievement goal research in sport: A response to Harwood, Hardy, and Swain. Journal of Sport and Exercise Psychology, 23, 317-29.

Willians, L. \& DeSteno. D. (2008). Pride and Perseverance: The Motivational Role of Pride. Journal of Personality and Social Psychology, 94(6), 1007-17.
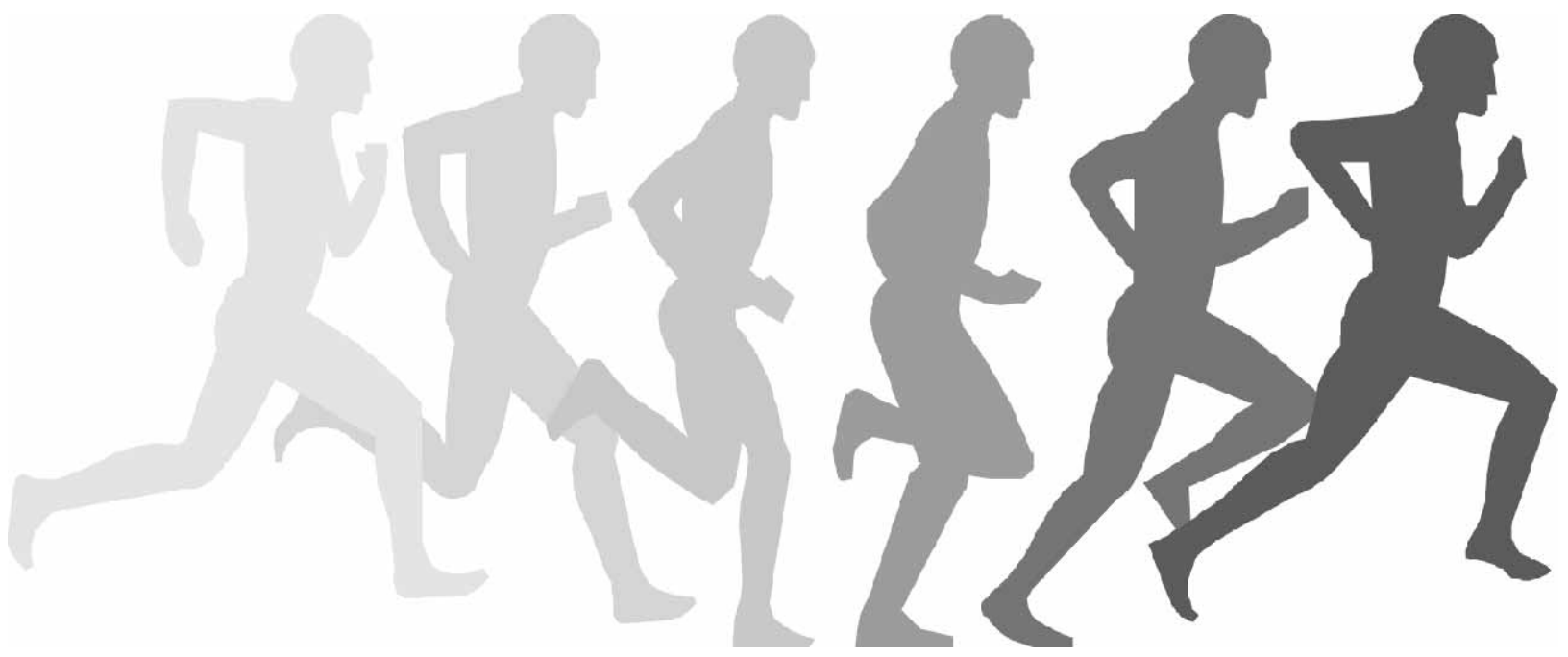